\title{
Cervical Crohn's disease with oesophago-pulmonary fistula
}

\author{
A. Steel, ${ }^{1}$ N.H. Dyer ${ }^{2}$ and H.R. Matthews ${ }^{1}$ \\ ${ }^{1}$ East Birmingham Hospital, Bordesley Green, Birmingham B9 5ST and ${ }^{2}$ Worcester Royal Infirmary, \\ Worcester, UK.
}

\begin{abstract}
Summary: We report what we believe to be the first case of Crohn's disease involving the cervical oesophagus with an oesophago-pulmonary fistula to the apex of the lung. The proximal location of the disease prevented radical therapy and the patient eventually died from chronic sepsis.
\end{abstract}

\section{Introduction}

Crohn's disease of the oesophagus with fistulae into the lung has been the subject of three previous case reports. All of these had involvement of the thoracic oesophagus. We report the first case of Crohn's disease of the cervical oesophagus with fistulae into the apex of the left upper lobe.

\section{Case report}

In 1973 a 45 year old male presented with subacute intestinal obstruction, thought to be due to Crohn's disease on barium follow-through examination. He subsequently developed an ileo-vesical fistula and underwent a right hemicolectomy and small bowel resection; histology revealed fissure formation and giant-cell granuloma formation, consistent with Crohn's disease. In 1974 he developed a right paracolic abscess and intestinal obstruction. Laparotomy revealed multiple adhesions with recurrence of Crohn's disease and a further resection of small and large bowel was performed. A year later he developed an abdominal wall abscess which was surgically drained, but sinuses persisted and oral prednisolone was commenced in 1978. In 1979 sinography showed that one sinus communicated with a segment of bowel. At laparotomy more small bowel was resected leaving only 3 feet of jejunum. Prednisolone was continued at a daily dose of $10-20 \mathrm{mg}$, with oral vitamin and medium chain triglyceride oil supplements.

For 6 years he remained in reasonable health. $\mathrm{He}$ then presented with a 6-month history of progressive dysphagia and pain on swallowing and a 2 -week history of coughing while drinking. He appeared grossly emaciated and dehydrated. He had a nor-

Correspondence: H.R. Matthews, F.R.C.S.

Accepted: 24 March 1988 mochromic normocytic anaemia, a neutrophil leucocytosis and an erythrocyte sedimentation rate of $125 \mathrm{~mm}$ in 1 hour. The serum phosphate was $0.60 \mathrm{mmol} / 1$ (normal range $0.80-1.40 \mathrm{mmol} / \mathrm{l}$ ), serum albumin was $16 \mathrm{~g} / \mathrm{l}$ and the serum alkaline phosphatase was raised at $497 \mathrm{U} / 1$ (normal range 30-130 U/1).

A chest radiograph showed a cavity in the left upper zone and bilateral old rib fractures (Figure 1). Barium swallow showed an irregular narrowing $6 \mathrm{~cm}$ long in the cervical oesophagus, radiating from which were multiple fistulae communicating with a left apical cavity and the trachea. Fibre-optie endoscopy revealed a stricture at $20 \mathrm{~cm}$ and a fistulous opening could be seen above the narrowing. Biopsies of the stricture were reported as showing 'acutely inflamed squamous epithelium and inflammatory debris'. A malignant process was suspected, however, and this was thought to be confirmed when an isotope bone scan showed numerous foci of increased uptake in the ribs, skull and pelvis, which were highly suggestive of metastatic disease. A fine bore nasogastric feeding tube was inserted and he was discharged.

Six months later he was still alive and although in poor health his condition had improved. He was referred to the Regional Thoracic Surgical Unit at East Birmingham Hospital for re-assessment of the diagnosis and possible treatment.

A further chest radiograph showed fibrosis at the site of the previous cavity (Figure 2). Barium swallow showed persistence of a 1-2 mm fistula between the oesophagus and the trachea. Rigid oesophagoscopy confirmed the presence of a stricture at $20-23 \mathrm{~cm}$, which was dilated to $54 \mathrm{FG}$. Biopsies of this showed evidence of chronic oesophagitis with no giant cell granulomata but were considered compatible with a diagnosis of Crohn's disease. No acid- and alcohol-fast bacilli or actinomycetes were subsequently isolated from his spu-

(C) The Fellowship of Postgraduate Medicine, 1988 


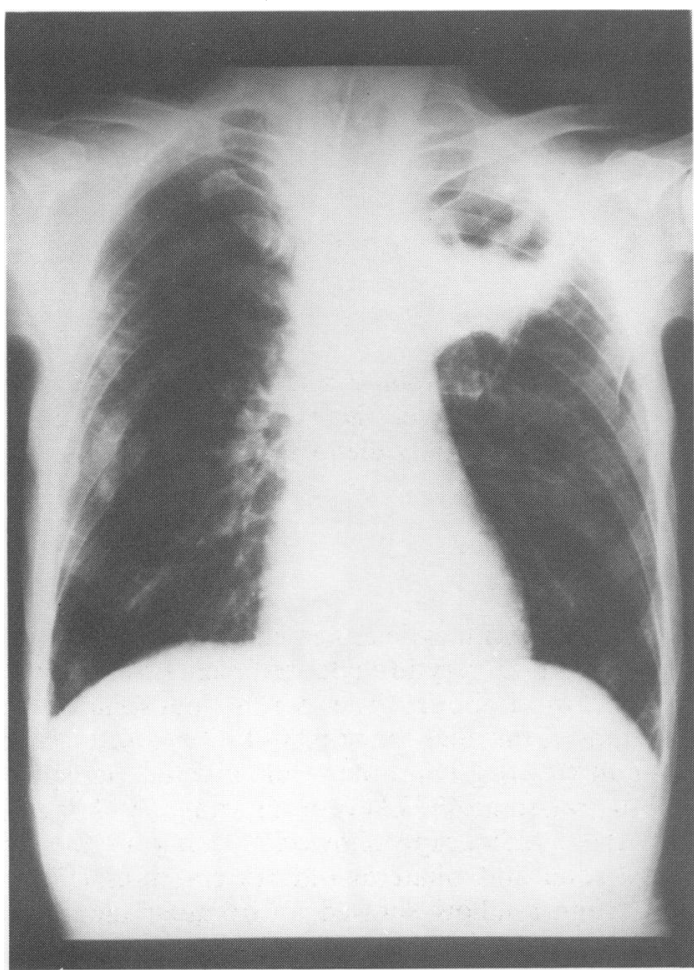

Figure 1 Chest radiograph showing left apical abscess.

tum. A diagnosis of Crohn's disease of the oesophagus was made.

The abnormalities on the bone scan were thought to be due to a combination of osteomalacia and osteoporotic rib fractures, possibly secondary to long-term steroid therapy. As the apical fistula appeared to have healed he was cautiously started on solids and then liquids, and was discharged home on an oral diet (with the nasogastric tube in situ for supplementary nutrition), oral prednisolone and fat soluble vitamins.

A month later he was re-admitted with a chest infection and recurrence of coughing while drinking. A Gastrograffin swallow showed a large fistula (Figure 3) between the oesophagus and a new cavity at the apex of the left hemithorax which communicated with a peripheral bronchus. An intercostal drain was inserted into the cavity and, in an attempt to close the fistula, an endo-oesophageal tube was inserted at endoscopy. However, he subsequently deteriorated and died 3 weeks later.

At autopsy the oesophagus was fibrotic with multiple fistulae arising from it extending into the trachea and both lung apices especially on the left (Figure 4). The apex of the left upper lobe was

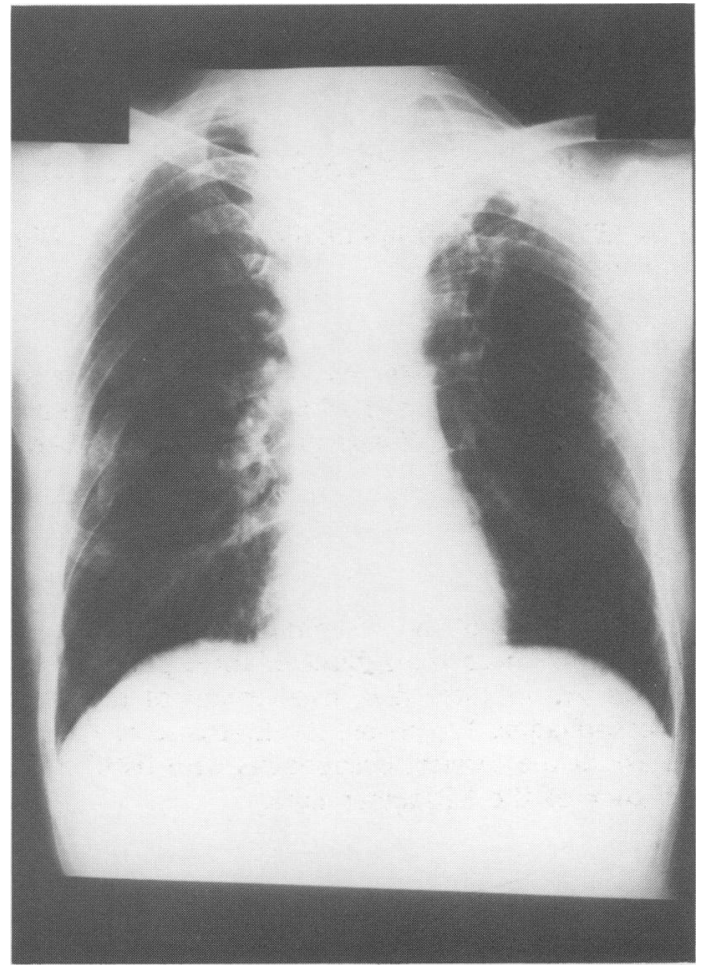

Figure 2 Chest radiograph showing fibrosis at site of previous abscess.

replaced by an $8 \mathrm{~cm}$ cavity which had connections with the smaller distal airways and the oesophagus. There were extensive adhesions within the abdominal cavity, with $120 \mathrm{~cm}$ remaining of the small bowel and $150 \mathrm{~cm}$ of the large bowel. Histological examination of the oesophagus showed extensive fibrosis of the submucosa and a chronic inflammatory cell infiltrate extending through the oesophageal wall with neuronal hyperplasia, consistent with Crohn's disease. Despite extensive examination of the sections no granulomata were seen.

\section{Discussion}

The first case of Crohn's disease involving the oesophagus was reported by Eggers in $1935,{ }^{1} 3$ years after Crohn's classic paper on ileal disease. ${ }^{2}$ Since then there have been a further 58 cases reported, including our patient. Of these 59 cases, the mean age at presentation with oesophageal symptoms was 39.9 years (range 12-73y) and 30 were male. Twenty-seven patients gave a previous history of Crohn's disease, 10 had oesophageal involvement as part of the initial attack, four 


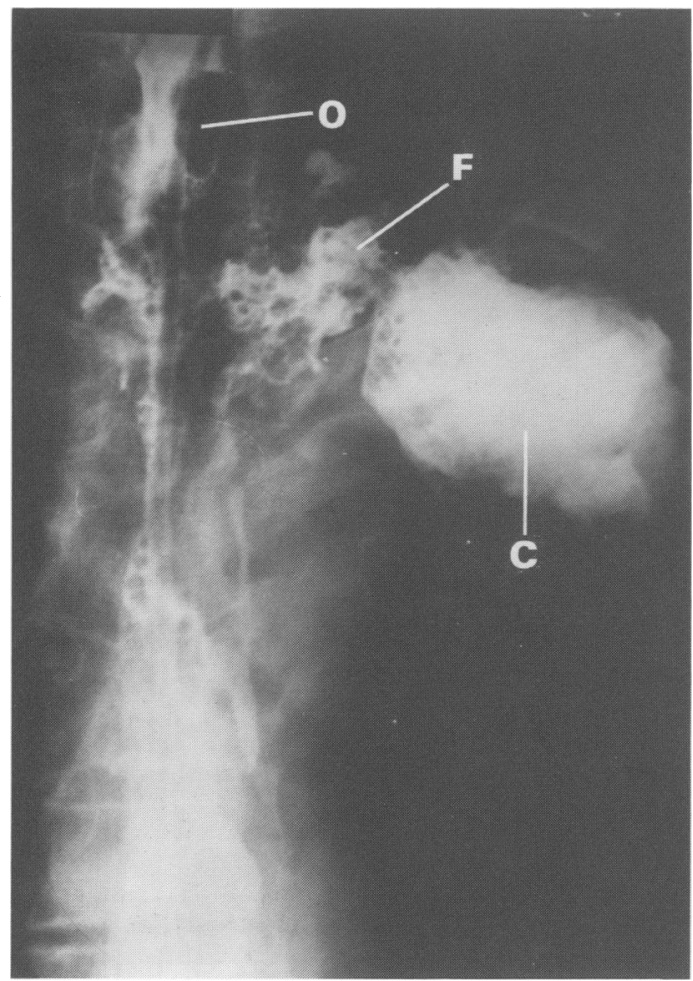

Figure 3 Barium swallow showing fistula $(F)$ between the oesophagus (O) and a large left apical cavity (C).

subsequently developed more classic Crohn's disease and in 15 patients the oesophagus appeared to be the only site of the disease, although clinical details in some reports were sparse.*

All patients with oesophago-pulmonary fistulae $^{3-5}$ had a history of Crohn's disease. In the first case $^{3}$ a 25 year old man gave a 9-month history of recurrent peri-anal sepsis, erythema nodosum and arthritis prior to the development of dysphagia. The second case, a 34 year old male, ${ }^{4}$ and the third case, a 50 year old female, ${ }^{5}$ gave a history of intraabdominal Crohn's disease over 17 and 14 years respectively, both having had intestinal resections. Our patient had a 12-year history of abdominal Crohn's disease and several operations. In the first case the fistulae and right upper lobe abscess developed 8 months after the dysphagia; in the second case 7 months separated the dysphagia and right-sided oesophago-bronchial fistula formation; in the third case a fistula and left lower lobe lung abscess formed 6 months after the onset of dysphagia. Our patient also had a 6-month history of

${ }^{*}$ A full list of references is available from the authors.

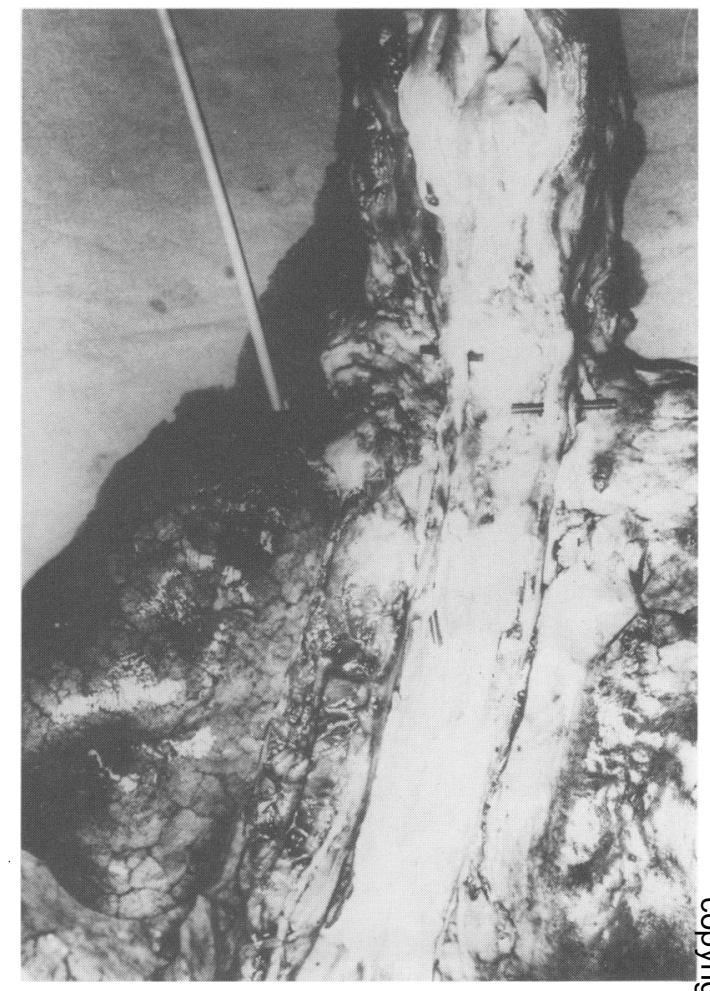

Figure 4 Post-mortem oesophagus viewed from the rear showing ulceration, thickened walls and bilateral fistulae (indicated by rods).

dysphagia prior to the development of fistulae and a left upper lobe abscess.

The first patient ${ }^{3}$ underwent oesophageal resection, the oesophagus from the cardia to below the clavicle being removed through a right sided approach. Although his recovery was slow the operation was successful and his associated conditions regressed. The second case $^{4}$ succumbed to lung sepsis and the third ${ }^{5}$ had a by-pass procedure with the stomach being anastomosed to the cervical oesophagus. This patient was reported to be well 9 months after her surgery. Only in the first case were giant cells seen in relation to a para-oesophageal tract, in the other two the histological changes were of a chronic inflammatory process with fibrosis consistent with Crohn's disease. Geboes et al. ${ }^{6}$ N emphasize that biopsies have to be subjected to close microscopic scrutiny if diagnostic evidence of Crohn's disease is to be found. They endoscoped 107 patients with upper gastrointestinal symptoms (95 patients) or stomatitis (12 patients) out of 500 Crohn's disease out-patients, of whom nine $(1.8 \%)$ had oesophageal lesions due to Crohn's disease. More often than not endoscopic biopsies showed 
non-specific chronic inflammatory changes within the mucosa, whereas in gross resected specimens the chronic inflammatory cell infiltrate is seen to extend through all layers of the oesophagus, often with fibrosis within the deeper layers.

The treatment of oesophageal involvement depends upon the degree of fibrosis. In the earliest stages of the disease, barium studies show only minor abnormalities, such as thickened mucosal folds or aphthous ulcers. ${ }^{7}$ Endoscopy may reveal aphthous ulcers or areas of erythema. These changes are often subtle and may be missed. At this stage of the disease if the diagnosis is made then medical management with corticosteroids and/or sulphasalazine is likely to be successful. ${ }^{6}$

The patient in this report was referred at a late stage of his disease. Because the cervical oesophagus was diseased a resection or by-pass procedure was not considered feasible. Treatment with

\section{References}

1. Eggers, C. Resection of thoracic portion of oesophagus for chronic ulcer. Ann Surg 1935, 101: 940-944.

2. Crohn, B.B., Ginzburg, L. \& Oppenheimer, G.D. Regional ileitis: a pathologic and clinical entity. JAMA 1932, 99: 1323-1329.

3. Achenbach, H., Lynch, J.P. \& Dwight, R.W. Idiopathic ulcerative oesophagitis. $N$ Engl J Med 1956, 255: 456-459.

4. Cynn, W., Chon, H. Gureghian, P.A. \& Levin, B.L. Crohn's disease of the oesophagus. Am J Roentgenol 1975, 125: 359-364. steroids and 'oesophageal rest' was partially successful but intubation failed to seal off the fistulae and he died of pulmonary sepsis and malnutrition.

Crohn's disease of the oesophagus is a recognized entity with a significant mortality and morbidity especially when complicated by fistulae. As the earlier stages of oesophageal involvement are amenable to treatment and in view of the rapid progression from initial dysphagia to life-threatening pulmonary complications, all patients with known Crohn's disease who develop dysphagia, and possibly stomatitis, should be investigated, initially by double contrast barium swallow and careful endoscopy, with multiple biopsies of any abnormal areas. Histological examination of the biopsies should involve multiple sections. If these tests fail to demonstrate evidence of Crohn's disease and symptoms persist then treatment with corticosteroids and/or sulphasalazine should be considered.

5. Ghahremani, G.G., Gore, R.M., Breuer, R.I. \& Larson, R.H. Oesophageal manifestations of Crohn's disease. Gastrointest Radiol 1982, 7: 199-203.

6. Geboes, K., Janssens, J., Rutgeerts, P. \& Vantrappen, G. Crohn's disease of the oesophagus. J Clin Gastroenterol 1986, 8: 31-37.

7. Gohel, V., Long, B.W. \& Richter, G. Aphthous ulcers in the oesophagus with Crohn's colitis. Am J Roentgenol 1981, 137: 872-873. 